\title{
Analysis of archival EXOSAT data on the X-ray burst source Serpens X-1
}

\author{
K.-I. Seon ${ }^{1}$ and K. W. Min ${ }^{2}$ \\ 1 Space Science Research Group, Korea Astronomy Observatory, 61-1 Whaam-dong, Yusong-gu, \\ Daejon 305-248, Korea \\ 2 Department of Physics, Korea Advanced Institute of Science and Technology, 373-1 Gusong-dong, Yusong-gu, \\ Daejon 305-701, Korea
}

Received 4 February 2002 / Accepted 22 August 2002

\begin{abstract}
We present analysis of data obtained from the Medium Energy proportional counter array (ME) from the EXOSAT observations of the X-ray burst source Ser X-1 made in 1983 and 1985. From the combined analysis of the spectral and fast variability properties, we show that the source is an atoll source in the banana state during the observations. The persistent spectra of the 1985 data, for which reliable background data are available, are described well by the two-component model composed of a power-law with an exponential cutoff and a blackbody. The spectral study of two bursts, which were observed during the 1985 observation, shows no clear evidence for a departure from blackbody. One of the bursts shows a weak secondary maximum, which is more prominent in the low energy band than in the high energy band, and the burst shows possible evidence of an absorption line feature at about $4.1 \mathrm{keV}$.
\end{abstract}

Key words. X-rays: stars - stars: individual: Ser X-1 - stars: neutron - X-rays: bursts

\section{Introduction}

Since a discovery in 1965 (Friedman et al. 1967), Ser X-1 (XB 1837+049) has been observed many times. As a result, Ser X-1 is now known to be relatively bright X-ray source (Forman et al. 1978; Priedhorsky \& Terrell 1984), with an estimated distance of about $7 \mathrm{kpc}$ (van Paradijs 1978). X-ray bursts from Ser X-1 were first detected in 1975 with the $O S O-8$ satellite within a circle of $5^{\circ}$ radius containing the source (Swank et al. 1976). A coincident X-ray and optical burst was detected in 1978 during several campaigns of coordinated optical and X-ray observations of burst sources (Hackwell et al. 1979). The optical burst was delayed by $\sim 1.4 \mathrm{~s}$ relative to the X-ray burst.

A detailed analysis of 57 bursts observed with $S A S-3$ in the period 1976 July to 1979 March was made by Sztajno et al. (1983). During this period, the persistent flux of Ser X-1 varied by a factor $\sim 2$ around an average value of $\sim 6 \times 10^{-9} \mathrm{erg} \mathrm{cm}^{-2} \mathrm{~s}^{-1}$ on the time scale of days. The occurrence of the bursts was so erratic that the burst frequency did not correlate with persistent flux or the burst flux. Spectral studies of the bursts from the source Ser X-1 detected by $S A S$ 3 were reported by Sztajno \& Bałucińska (1983) and also by Bałucińska \& Czerny (1985).

Ser X-1 was also observed with the Einstein (Vrtilek et al. 1986, 1991; Lum et al. 1992). Vrtilek et al. (1986) found a spectral hardening with an increase in luminosity. EXOSAT

Send offprint requests to: K.-I. Seon, e-mail: kiseon@kao.re.kr also observed the source twice in 1983 and 1985. Schulz et al. (1989) found a positive correlation in the color-color diagram using the ME data from EXOSAT observations of 1983 and 1985. White et al. (1986) and Gottwald et al. (1995) analysed the spectrum of the persistent emission using Gas Scintillation Proportional Counter (GSPC) data and reported that a single-component model can give an acceptable fit. They also observed an iron line at $\sim 6.4-6.5 \mathrm{keV}$.

More recently, ASCA observed the source in 1994 (Church \& Bałucińska-Church 2001; Asai et al. 2000), and BeppoSAX and RXTE simultaneously in 1999 (Oosterbroek et al. 2001). Unfortunately, the source did not show any burst activity during the observations. Furthermore, Schulz et al. (1989) wrongly stated that some burst activity could be observed during the first EXOSAT observation period, while the second observation did not show burst activity. The present analysis shows clearly burst activity during both observations.

The continuum spectrum of X-ray bursts resembles a cooling blackbody, from which the radius of a neutron star can be estimated, although there is evidence for modification of the blackbody in some bursts. Discrete spectral features may reveal the redshift of neutron star surface and so constrain the mass-radius relation of the neutron stars. Since the discovery of absorption features in XB 1636-536 by Waki et al. (1984), the existence of the absorption lines in four X-ray burst sources has been reported by several authors (see, e.g. Madej 1990; Asai et al. 1998). The line features are usually interpreted as the 


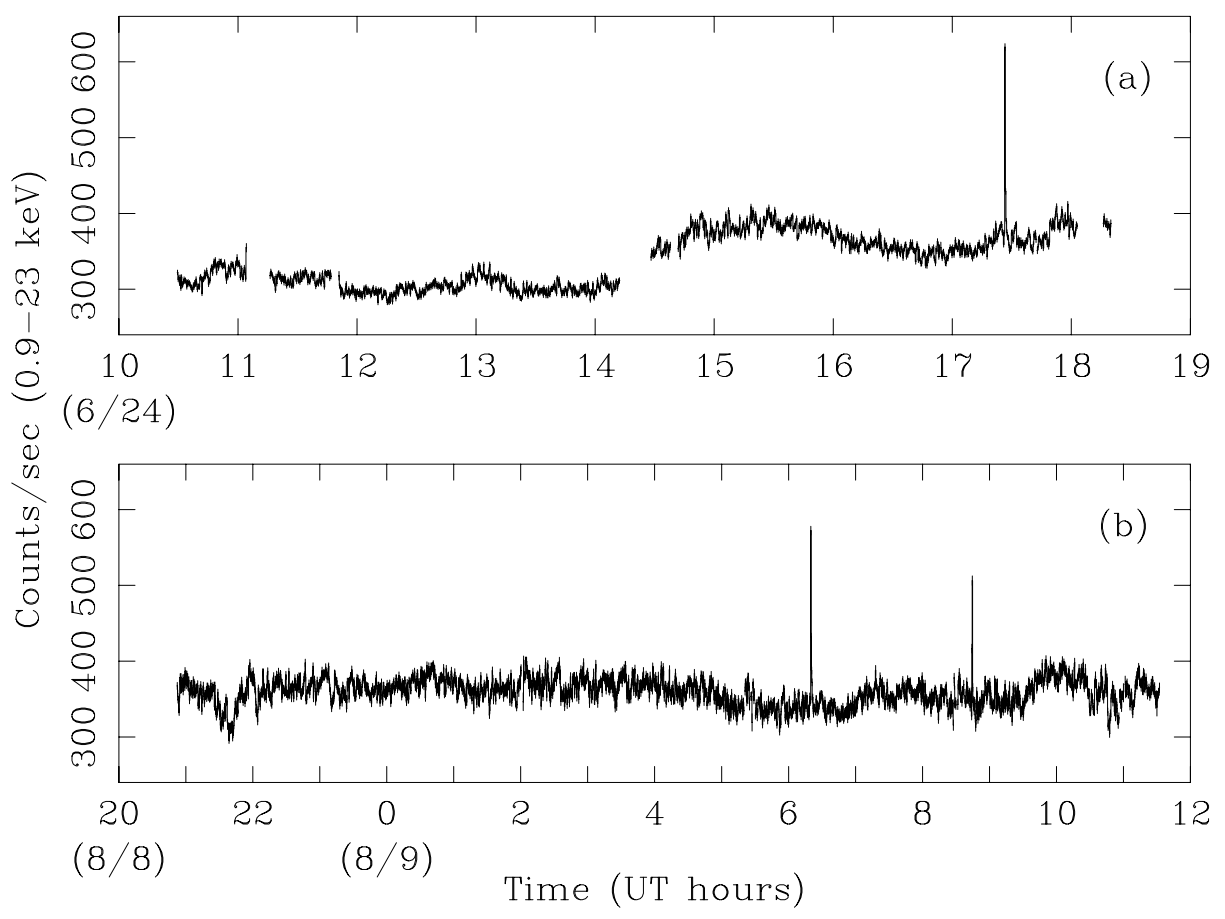

Fig. 1. The light curve of Ser X-1 in the energy band $0.9-23 \mathrm{keV}$ obtained a) at 1983 July 24 and b) at 1985 August 8 . The data were deadtime corrected and background subtracted. Each data point is an accumulation for $20 \mathrm{~s}$.

gravitationally redshifted resonance line of helium-like ions of a heavy element. We also found marginal evidence of the absorption line feature at $\sim 4.1 \mathrm{keV}$ from a burst spectrum at the secondary maximum of a double-peaked burst.

In spite of the importance of the burst activity of Ser X-1, in terms of its relative brightness among burst sources, the burst analyses for the source had been done using rather older SAS-3 observations. It is also important to study as many timeresolved burst spectra as possible, to search for an absorption line, and to obtain an understanding of such a feature. This motivated the present analysis of archival EXOSAT data. In this study we report results of the detailed analysis of the two EXOSAT observations of Ser X-1 made by ME in 1983 and 1985, which were overlooked in the previous studies.

\section{Observations}

The burst source Ser X-1 was observed with EXOSAT from 1983 July 24, 8:50 UT to July 24, 18:59 UT, and from 1985 August 8, 20:11 UT to August 9, 13:40 UT. The data presented here are obtained from the argon layer of the ME experiment (Turner et al. 1981). The observation in 1983 was made during the performance verification (PV) phase, with the high energy resolution mode HER2, in which the whole array of eight detectors were pointed at the source. There are no background data for the 1983 observation. During the 1985 observation the data were obtained in the high energy resolution mode HER5, in which four detectors of the half array pointed at Ser X-1 and the other half array was offset by $\sim 2^{\circ}$ to monitor the background. The background was very stable during the 1985 observation of Ser X-1. To reduce the systematic error in the observation each half of the detector array was alternately offset to a source-free region of the sky and the background was obtained from the offset spectra of the same half and corrected for the known offset-angle dependence. However, one of four detectors in the half array failed at the time of 1985 observation. Pulse height analysis data were obtained in 125 channels with a time resolution of $0.531 \mathrm{~s}$ and $0.625 \mathrm{~s}$ for the 1983 observation, and in 64 channels with a time resolution of $1 \mathrm{~s}$ for the 1985 observation. The high time resolution data HTR3, with the timing resolution of $31.25 \mathrm{~ms}$, is available only for the 1985 observation.

\section{Analysis and results}

\subsection{Persistent emission}

The light curves obtained from the 1983 observation and the 1985 observation are shown in Fig. 1; three bursts are clearly visible in the figure which were overlooked in the previous analyses. Each point in the figure represents the data averaged over $20 \mathrm{~s}$. All counting rates quoted are in units of counts/s per half array. Since the background information is not available for the 1983 observation, we used the combination of the background data obtained from the both halves of the detector in 1985 observation, as was done by Schulz et al. (1989). The total count rates during the 1983 and 1985 observations, except during the bursts, vary from $\sim 280$ to $\sim 370$ counts/s and from $\sim 300$ to $\sim 400$ counts/s, respectively, in the energy band $0.9-23 \mathrm{keV}$. The 1983 data in Fig. 1a shows several gaps. The reason for these gaps is not certain. The count rate in the second half of the data is higher than the first half before the gap near 14:20 UT. The GSPC data shows a similar trend and gaps in the count rate as the ME data. The source is $\sim 12 \%$ brighter during the 1985 observation on the average than during the 1983 observation. 


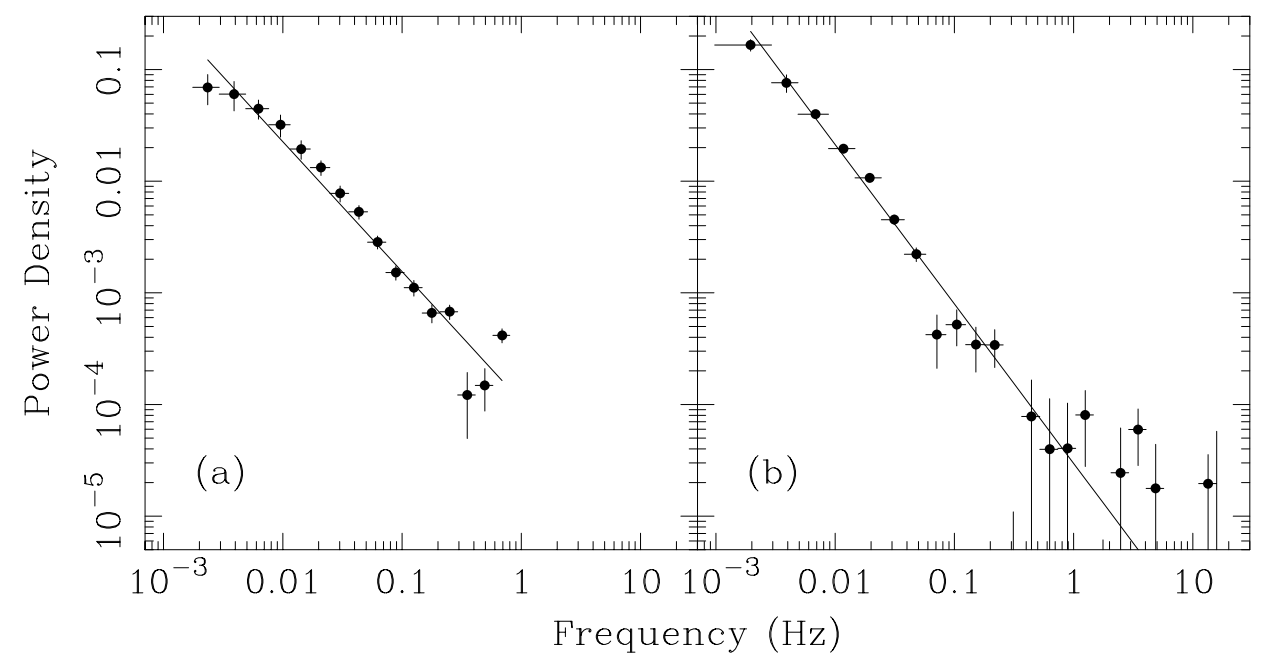

Fig. 2. Power spectra obtained a) from the 1983 observation and b) from the 1985 observation. The drawn lines represent the best fit power law model. Note a possible flattening above $1 \mathrm{~Hz}$ during the 1985 observation.

Color-color and intensity-color diagrams were also examined. The color-color diagram shows a slight positive correlation in both observations, which is typical of an atoll source in the banana state (Hasinger \& van der Klis 1989), and the positive correlation in both observations are consistent with the results of Schulz et al. (1989).

The power spectra obtained using the 1983 data with a time resolution of $0.625 \mathrm{~s}$ and the 1985 data with $31.25 \mathrm{~ms}$ time resolution are shown in Figs. 2a and b. The data were divided into equal segments and each segment was transformed individually using a Fast Fourier Transform (FFT), and then the power spectra were averaged. In the averaged power spectra, 40 individual segments were used for the 1983 data and 104 for 1985 data. The resulting average power spectra were rebinned and the average level of the white noise was subtracted, while Poisson counting statistics were taken into account as modified by dead time effects (see, e.g. van der Klis 1989). The power spectra were fitted by a power-law, with a best fit value of the powerlaw index $\alpha=-1.16 \pm 0.05$. The fractional root-mean square (rms) variation integrated over $0.005-1 \mathrm{~Hz}$ is $3.0 \pm 0.2 \%$ for the 1983 observation. We could not check the presence of the noise above $1 \mathrm{~Hz}$ for this observation because of the lack of high time resolution data. The fractional $\mathrm{rms}$ variation integrated over the region $0.005-1 \mathrm{~Hz}$ of the 1985 data is $2.4 \pm 0.5 \%$ and the best fit value of the power-law index $(\alpha)$ is $-1.43 \pm 0.04$. The power spectra obtained from both observations are typical of an atoll source in the banana state (Hasinger \& van der Klis 1989). It seems there remains a flat component above $1 \mathrm{~Hz}$ during the 1985 observation, although the error bars are rather large. If this feature is taken to be real, it may suggest that the source was in the lower banana state at the time of 1985 observation.

The absence of reliable background data for the 1983 observation makes it difficult to analyse the spectrum in detail. Thus we concentrate only on the spectrum obtained from the 1985 observation. We tried several single component models as well as more complex two component models. The results and the reduced $\chi_{v}^{2}$-values are listed in Table 1 . As can be seen from the table, single component models do not describe the spectrum
Table 1. Reduced $\chi^{2}$ values for several models.

\begin{tabular}{lc}
\hline \hline Model & $\chi_{v}^{2}$ (d.o.f.) \\
\hline BREMSS $^{a}$ & $5.00(61)$ \\
PL $^{b}$ & $52.7(61)$ \\
CUTPL $^{c}$ & $1.81(60)$ \\
COMPST $^{d}$ & $3.22(60)$ \\
DISKBB $^{e}$ & $22.0(61)$ \\
BREMSS+BBODY $^{f}$ & $1.37(59)$ \\
DISKBB+BBODY & $2.38(59)$ \\
DISKBB+CMPB $^{g}$ & $1.67(59)$ \\
CUTPL+BBODY & $1.19(58)$ \\
COMPST+BBODY & $1.19(58)$ \\
CUTPL+BBODY+GAUSs $^{h}$ & $1.00(55)$ \\
COMPST+BBODY+GAUSS & $1.11(55)$ \\
\hline
\end{tabular}

${ }^{a}$ Thermal bremsstrahlung. ${ }^{b}$ Power-law. ${ }^{c}$ Power-law with exponential cutoff. ${ }^{d}$ Comptonization model (Sunyaev \& Titarchuk 1980). ${ }^{e}$ Disk blackbody. ${ }^{f}$ Blackbody. ${ }^{g}$ Comptonized blackbody (Nishimura et al. 1986). ${ }^{h}$ Gaussian line.

well, while two-component models generally give acceptable fits, except the ones involving a disk blackbody. Table 2 shows the best fit values when the combination of a power-law with an exponential cutoff and a blackbody is used. Since residuals still show a line feature around $\sim 6.8 \mathrm{keV}$, we have included the Gaussian line representing the iron emission line and the goodness of fit improved. The flux obtained from this model is $\sim 7 \times 10^{-9} \mathrm{erg} \mathrm{cm}^{-2} \mathrm{~s}^{-1}$ in the energy band $1-20 \mathrm{keV}$, corresponding to the luminosity $L \simeq 4.1 \times 10^{37} \mathrm{erg} \mathrm{s}^{-1}$ assuming a distance of $7 \mathrm{kpc}$. The blackbody component contributes $\sim 10$ $17 \%$ to the total flux. The unfolded spectrum and the model are plotted in Fig. 3. 
Table 2. Best-fit parameters for the persistent spectra.

\begin{tabular}{lcc}
\hline \hline Parameters & CUTPL+BBody & cutPl+Bbody+GAuss \\
\hline$F_{\text {cutpl }}{ }^{a}\left(10^{-9} \mathrm{erg} \mathrm{cm}^{-2} \mathrm{~s}^{-1}\right)$ & $5.8_{-0.8}^{+1.0}$ & $5.9_{-0.9}^{+1.1}$ \\
$\alpha^{b}$ & $1.8_{-0.3}^{+0.2}$ & $1.6_{-0.3}^{+0.3}$ \\
$E_{\text {cut }}{ }^{c}(\mathrm{keV})$ & $11_{-3}^{+6}$ & $8_{-2}^{+3}$ \\
$F_{\text {bbody }}{ }^{a}\left(10^{-9} \mathrm{erg} \mathrm{cm}^{-2} \mathrm{~s}^{-1}\right)$ & $1.4_{-0.3}^{+0.2}$ & $0.9_{-0.4}^{+0.3}$ \\
$k T_{\mathrm{b}}{ }^{c}(\mathrm{keV})$ & $1.51_{-0.05}^{+0.04}$ & $1.46_{-0.11}^{+0.07}$ \\
$R_{\mathrm{b}}{ }^{d}(\mathrm{~km})$ & $3.5_{-0.6}^{+0.3}$ & $3.1_{-0.6}^{+0.4}$ \\
$E_{\text {line }}{ }^{c}(\mathrm{keV})$ & $\cdots$ & $6.8_{-0.5}^{+0.3}$ \\
$E W^{e}(\mathrm{eV})$ & $\ldots$ & $52_{-24}^{+25}$ \\
$N_{\mathrm{H}}{ }^{f}\left(10^{21} \mathrm{~cm}^{-2}\right)$ & $7.1_{-1.4}^{+1.3}$ & $5.9_{-1.4}^{+1.5}$ \\
$\chi_{v}^{2}($ d.o.f. $)$ & $1.2(58)$ & $1.0(56)$ \\
\hline
\end{tabular}

Note. Quoted errors are 90\% confidence limits.

a Fluxes of CutPL and BвоDY components, respectively, for the energy band $1-20 \mathrm{keV}$.

${ }^{b}$ Power-law index

c Blackbody temperature $k T_{\mathrm{b}}$, cutoff energy $E_{\text {cut }}$, and line energy $E_{\text {line }}$.

d Blackbody radius for an assumed distance $7 \mathrm{kpc}$.

$e$ Equivalent width.

$f$ Neutral hydrogen column density.

\subsection{Bursts}

The light curves of two bursts from 1985 observation, after being corrected for the dead time and the background, are shown in Fig. 4. The data were obtained from the timing program (HTR3) with a time resolution of $31.25 \mathrm{~ms}$. The count rate reached a maximum $\gtrsim 1300$ counts/s for both bursts. It is interesting to note that the second burst in Fig. 4b shows a secondary maximum about $\sim 6 \mathrm{~s}$ away from the main peak. In Fig. 5, we plotted the light curves in two energy bands (0.9-6 and $6-20 \mathrm{keV}$ ) for the second burst. The figure shows that the secondary maximum is visible in both energy bands, although it is more prominent in the low energy band than in the high energy band. This secondary peak appears only in one time bin because of the low time resolution (1 s) of the spectral data.

In Table 3 we list some basic characteristics of the bursts: the burst fluence $\left(E_{\mathrm{b}}\right)$, burst peak flux $\left(F_{\max }\right)$, the burst rise time and decay time, time elapsed since the preceding burst $(\Delta t)$, the mean persistent flux during that interval $\left(F_{\mathrm{p}}\right)$, the ratio $\alpha$ of the fluence of the persistent emission to the burst fluence $\left(F_{\mathrm{p}} \Delta t / E_{\mathrm{b}}\right)$, and the burst duration $\tau$ given by $E_{\mathrm{b}} / F_{\max }$. The burst rise time is defined as the time interval during which the observed count rate increases to the peak flux. The decay time is defined as the e-folding time for which the count rate decreases from its maximum.

The time-resolved burst spectra obtained from the $1985 \mathrm{ob}-$ servation were studied in detail using several spectral models. It was pointed out by van Paradijs \& Lewin (1986) that a standard analysis of X-ray burst spectra, in which a Planck function is fitted to the net burst emission, will give rise to systematic errors in the temperature and the radius determination. This effect was found to be important in a burst observed from the

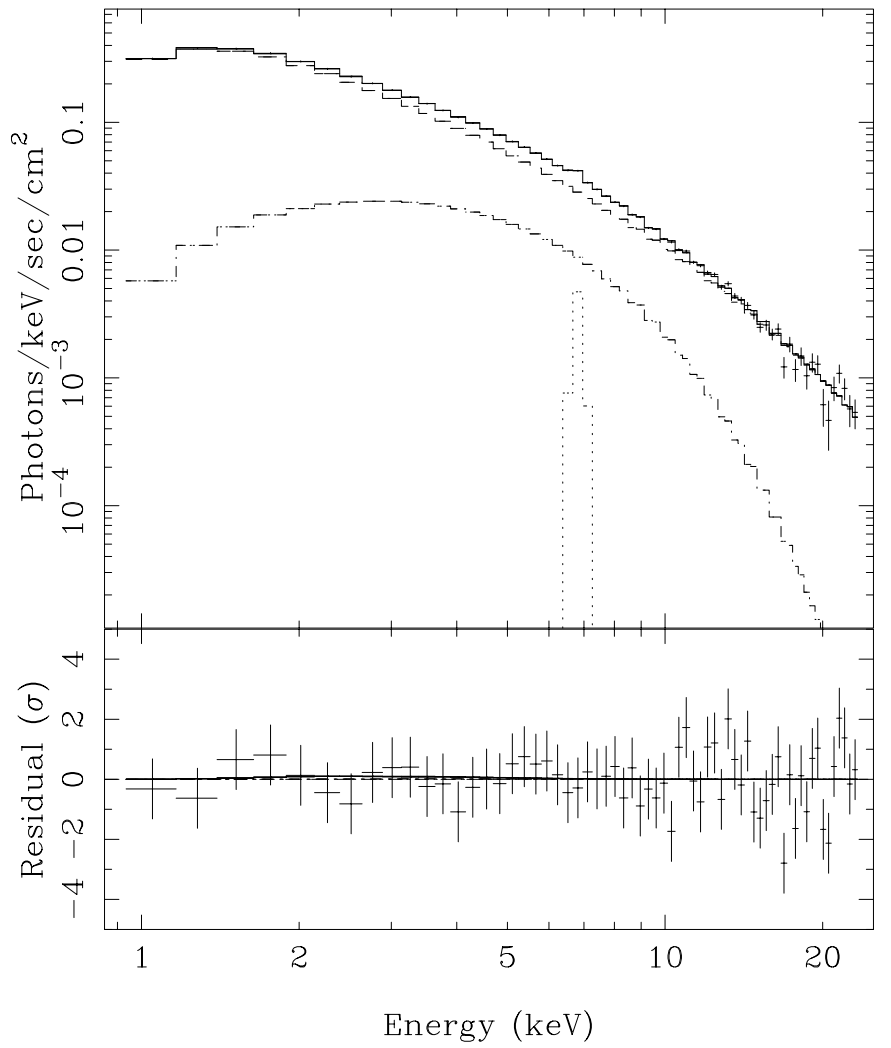

Fig. 3. Unfolded energy spectrum and the best fit model for the 1985 observation. The continuum model is cuTPL+BBoDy. See Table 2 for best-fit parameters.

Table 3. Properties of the bursts of Ser X-1.

\begin{tabular}{lccc}
\hline \hline Start time & Aug. 8 1983 July 24 1985 & July 24 1985 \\
$(\mathrm{UT})$ & $17: 26: 25$ & $06: 19: 49$ & $08: 44: 25$ \\
\hline$E_{\mathrm{b}}{ }^{a}\left(10^{-8} \mathrm{erg} \mathrm{cm}^{-2}\right)$ & $\ldots$ & $7.8 \pm 0.3$ & $8.0 \pm 0.4$ \\
$F_{\max }{ }^{b}\left(10^{-8} \mathrm{erg} \mathrm{cm}^{-2} \mathrm{~s}^{-1}\right)$ & $\ldots$ & $1.5 \pm 0.1$ & $1.9 \pm 0.2$ \\
rise time (s) & $0.9 \pm 0.6$ & $0.4 \pm 0.1$ & $0.30 \pm 0.06$ \\
decay time (s) & $5.3 \pm 0.2$ & $5.0 \pm 0.3$ & $3.3 \pm 0.3$ \\
$\Delta t^{c}(\mathrm{~h})$ & $>6.95$ & $>9.46$ & 2.41 \\
$F_{\mathrm{p}}{ }^{d}\left(10^{-9} \mathrm{erg} \mathrm{cm}^{-2} \mathrm{~s}^{-1}\right)$ & $\ldots$ & 6.98 & 6.67 \\
$\alpha^{e}$ & $\ldots$ & $>3040$ & 730 \\
$\tau^{f}(\mathrm{~s})$ & $\ldots$ & $5.4 \pm 0.6$ & $4.2 \pm 0.4$ \\
\hline
\end{tabular}

Note. Quoted errors are $90 \%$ confidence limits.

a Burst fluence.

${ }^{b}$ Burst peak flux.

${ }^{c}$ Burst time interval since the preceding burst.

$d$ Mean persistent flux during the burst time interval $\Delta t$.

$e$ Ratio of the total energy emitted in the persistent X-ray emission to that emitted in the burst $\left(F_{\mathrm{p}} \Delta t / E_{\mathrm{b}}\right)$.

$f$ Burst duration $\left(E_{\mathrm{b}} / F_{\max }\right)$.

luminous source GX 17+2 (Sztajno et al. 1986). We therefore analysed the data in two different ways. First, we subtracted from the burst spectrum the persistent spectrum plus the background accumulated over $\sim 600 \mathrm{~s}$ just prior to the burst and fitted the net burst spectrum using a conventional blackbody 


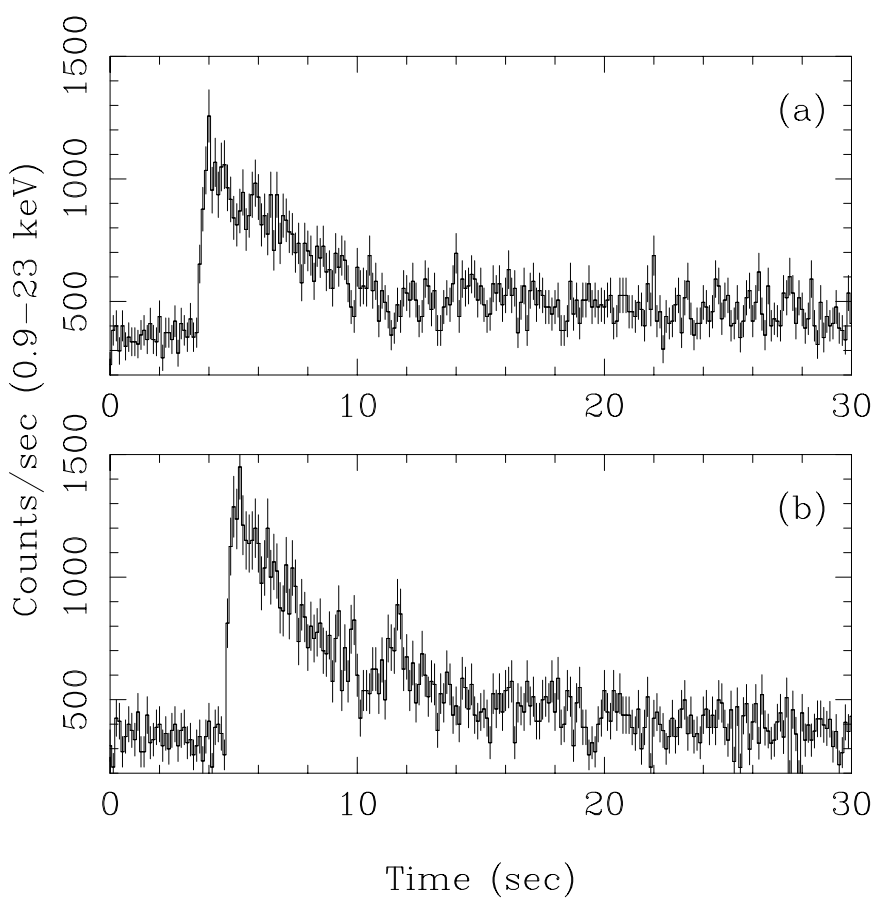

Fig. 4. Light curve a) of the first burst observed in 1985, and b) of the second burst observed in 1985. Each point represents an accumulation over $0.125 \mathrm{~s}$. Note that the second burst shows weak secondary maximum.

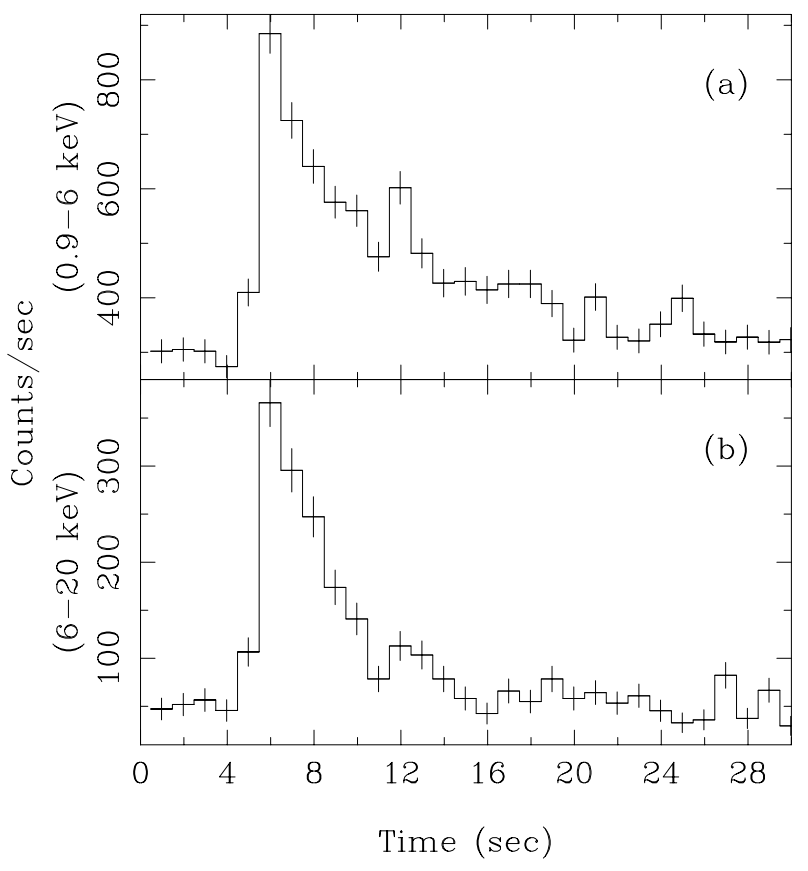

Fig. 5. Light curves in two energy bands, a) $0.9-6 \mathrm{keV}$, and b) 6$20 \mathrm{keV}$ for the second burst observed in 1985 . Note a weak secondary maximum, which is more prominent in the low energy band than in the high energy band.

model. The burst spectra were accumulated over $1 \mathrm{~s}$ for each bin near the peak of the burst, and over $3 \mathrm{~s}$ in the tail. In the second method, following the suggestion of van Paradijs \& Lewin (1986), we fitted the spectrum including both burst and persistent emissions by the two-component model consisting of a

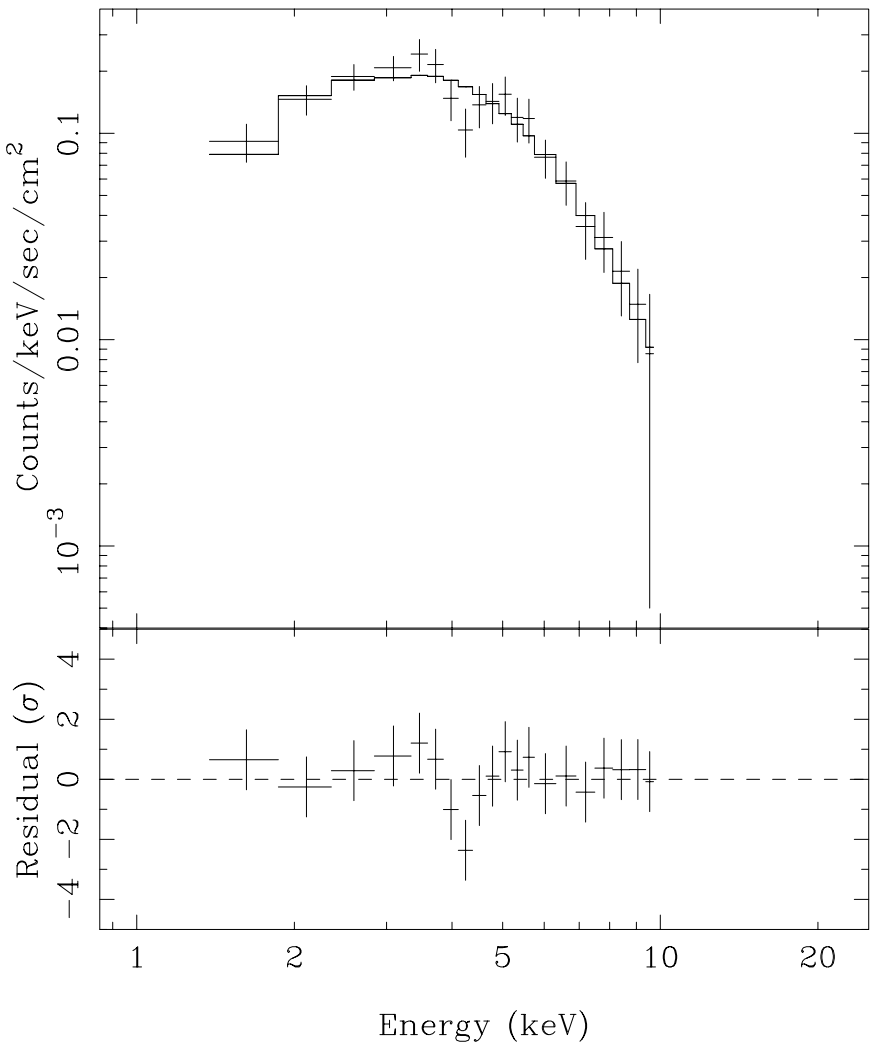

Fig. 6. A discrete spectral feature around $4.1 \mathrm{keV}$, observed at the secondary maximum of the second burst in the 1985 observation.

variable blackbody ввоDY and a fixed disk component CUTPL. The data were accumulated over $1 \mathrm{~s}$ for each bin in this case. The statistical quality of the data is not good enough for an accurate determination of $N_{\mathrm{H}}$ in both cases. We therefore used the $N_{\mathrm{H}}$ value obtained from the spectral fits to the persistent $\mathrm{X}$-ray emission.

Most of the reduced $\chi^{2}$ values obtained from the burst spectral analysis were acceptable (they varied between 0.6-1.5). We found some evidence for a discrete feature at only one occasion, at the secondary maximum of the second burst observed in 1985. Figure 6 shows the burst spectrum of the second burst and the best fit model following the second method. The figure suggest that there is a systematic deviation near $4 \mathrm{keV}$ from the best fit model, although the model gives an acceptable $\chi^{2}$ value. We therefore tried to fit this feature using an absorption line. The line energy and the equivalent width of the absorption line were $4.2 \pm 0.4 \mathrm{keV}$ and $380 \pm 260 \mathrm{eV}$, respectively, at $90 \%$ level of confidence. The $\chi^{2}$ values of the fit without an absorption line was 12.0 for 18 d.o.f., and that with an absorption line was 7.2 for 16 d.o.f. An $F$-test indicates a probability of this occurring if the absorption feature is not real of less than $1.6 \%$. Although the detection of the absorption line is marginal, it is worth noting that the central energy of the absorption line is close to that of the absorption lines found from the other sources (Waki et al. 1984 for XB 1636-536 and Nakamura et al. 1988 for XB 1608-522).

Figures 7 to 9 depict various results of the blackbody component obtained for the two bursts in 1985 obtained by two different methods. In Fig. 7, the bolometric flux, color 


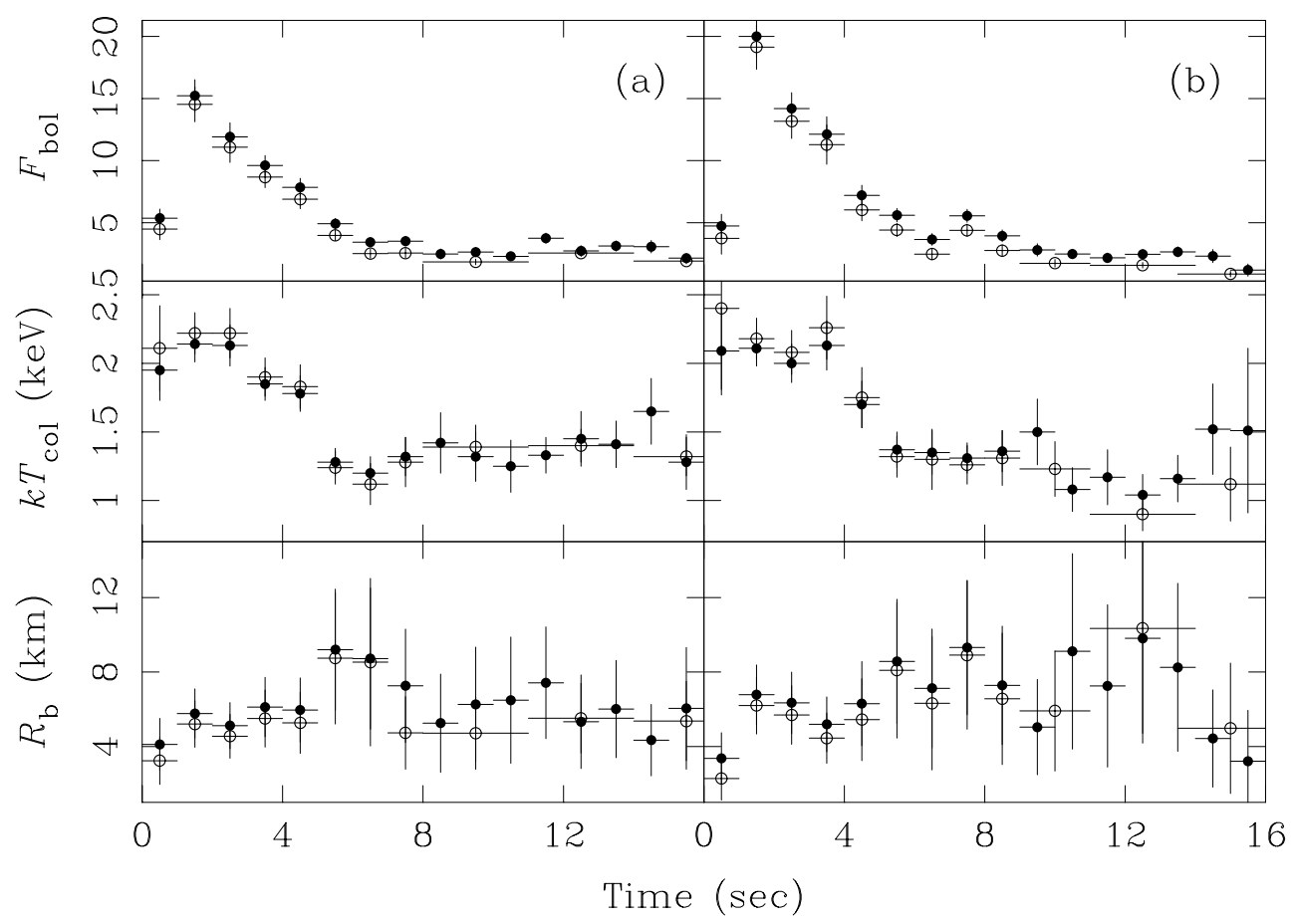

Fig. 7. Bolometric flux, color temperature, and blackbody radius as a function of time a) for the first burst and b) for the second burst of the 1985 observation. The bolometric flux is in units of $10^{-9} \mathrm{erg} \mathrm{cm}^{-2} \mathrm{~s}^{-1}$. Open circles represent the values obtained from the standard analysis, in which the net burst spectra are fitted by a blackbody. Closed circles are obtained from the spectral analysis of the total (burst and persistent) emission fitted by a two-component model consisting of a variable blackbody and a fixed disk component.

temperature $T_{\mathrm{col}}$ and the corresponding apparent blackbody radius $R_{\mathrm{b}}=\mathrm{d}\left(F_{\text {bol }} / \sigma T_{\text {col }}^{4}\right)^{1 / 2}$ are calculated by assuming that the emission is isotropic and the source is $7 \mathrm{kpc}$ away. The two methods give similar results, although the spectral analysis of the total (burst plus persistent) emission generally yields larger values for the bolometric flux and the blackbody radius, and smaller values for the color temperature than those obtained from the standard analysis. There is no evidence for the systematic decrease of the blackbody radius as the net burst flux goes to zero, an artificial effect found in the burst analysis from the luminous source GX 17+2 (Sztajno et al. 1986). The result indicates that the persistent blackbody component is small compared to the burst emission, which is indeed true in the present case. The sudden increase of the blackbody radius near $6 \mathrm{~s}$ in Fig. 7a is not associated with the real radius expansion usually seen at the peak of the burst, but it may be due to the inconsistency of the effective temperature with the color temperature. The anti-correlation between the blackbody radius and the color temperature may result from the same effects. However, the statistics of the present data are not good enough to give a decisive conclusion.

In Fig. $8, \log F_{\text {bol }}$ is plotted against $\log k T_{\text {col }}$ for the two bursts in 1985 . For a perfect blackbody with a constant radius the track should be on the straight line with a slope of 4 . When the data is fitted with a straight line taking into account the data errors in both $x$ and $y$, the slope is less than 4 with its value $3.1 \pm 1.0$ for the first burst and $3.2 \pm 0.9$ for the second burst. The straight lines with a slope of 4 are also shown as dashed lines in the figure for comparison. We also calculated a quantity $\theta \equiv k T_{\mathrm{col}} / F_{\mathrm{bol}}^{1 / 4}$, which is proportional to the ratio of color temperature to the effective temperature. In Fig. 9 the quantity $\theta$ is plotted as a function of $k T_{\text {col }}$; From the linear least-squares fits we obtain the slopes of $0.2 \pm 0.1$ for the first burst and $0.2 \pm 0.2$ for the second burst taking into account the data errors in both $x$ and $y$. The straight lines with a constant value are also shown as dashed lines in the figure for comparison. Although we analysed the data in two different methods, the results in Figs. 7 to 9 are similar regardless of the methods chosen for the analysis. The slopes found in Figs. 8 to 9 were obtained by adding closed circle and open circle data. The deviation of the burst spectra from a simple blackbody spectra shape cannot be shown conclusively, mainly due to insufficient data, although the results show some departure of the spectra from the simple blackbody shape.

\section{Discussion}

In this paper we studied the archival EXOSAT data of Ser X-1, which were not fully analysed in previous analyses. During both observations in 1983 and in 1985 the source showed behavior typical of an atoll source in the banana state, as the combined analysis of the color-color diagram and the power spectrum reveals.

White et al. (1988) extensively studied various types of two component models using EXOSAT ME observations and showed that the spectra of faint burst sources can be represented by a single component while the non-bursting bright sources can be understood in terms of two components. White et al. (1986) and Gottwald et al. (1995) adopted a simple one component model to represent the spectra of Ser X-1 obtained 


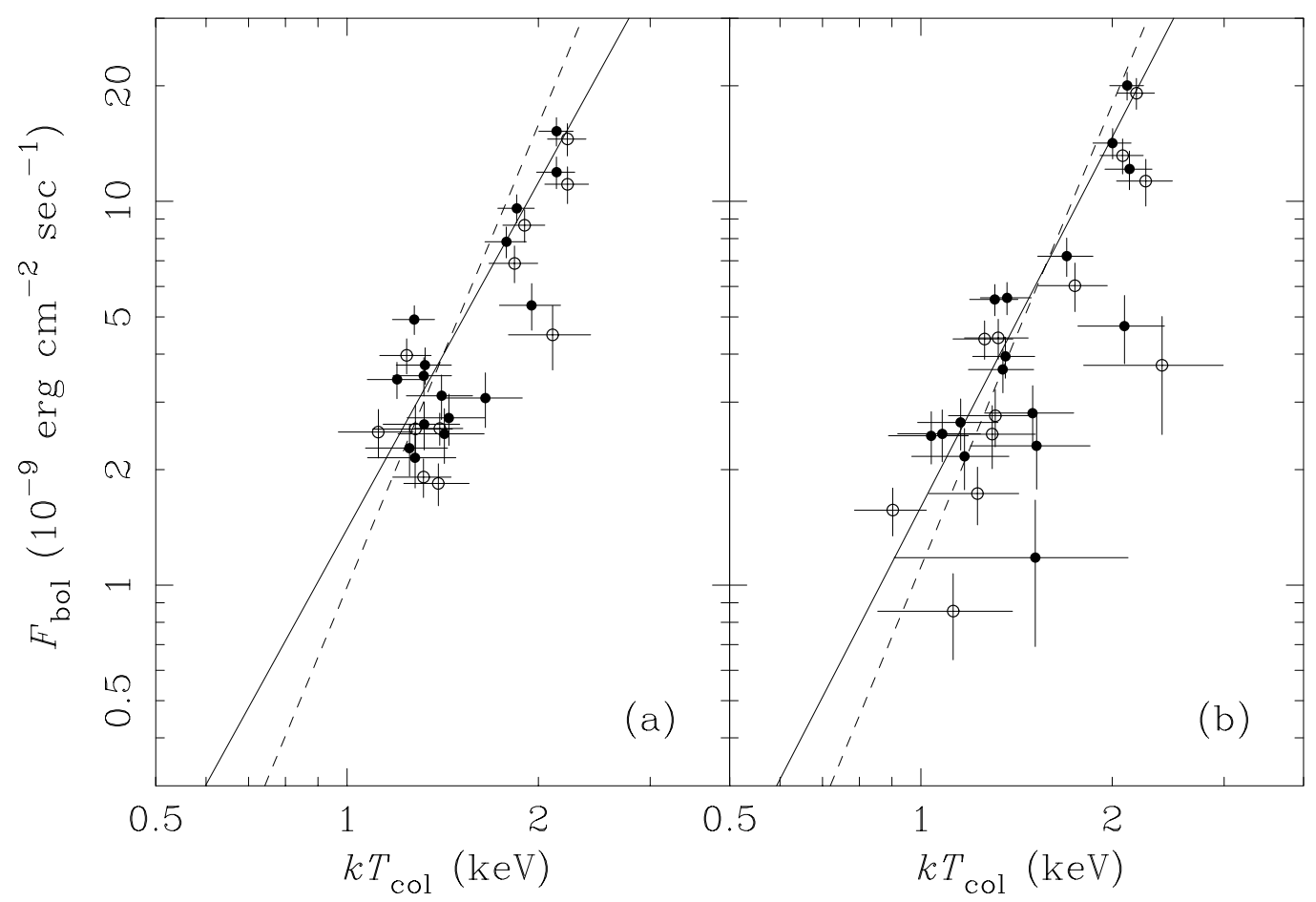

Fig. 8. The bolometric flux is plotted versus the observed color temperature a) for the first burst and b) for the second burst of the 1985 observation. Open circles and closed circles represent the values obtained by two different methods, as they do in Fig. 7. Best-fit straight lines are shown as solid lines and straight lines with a slope of 4 as dashed lines for comparison.

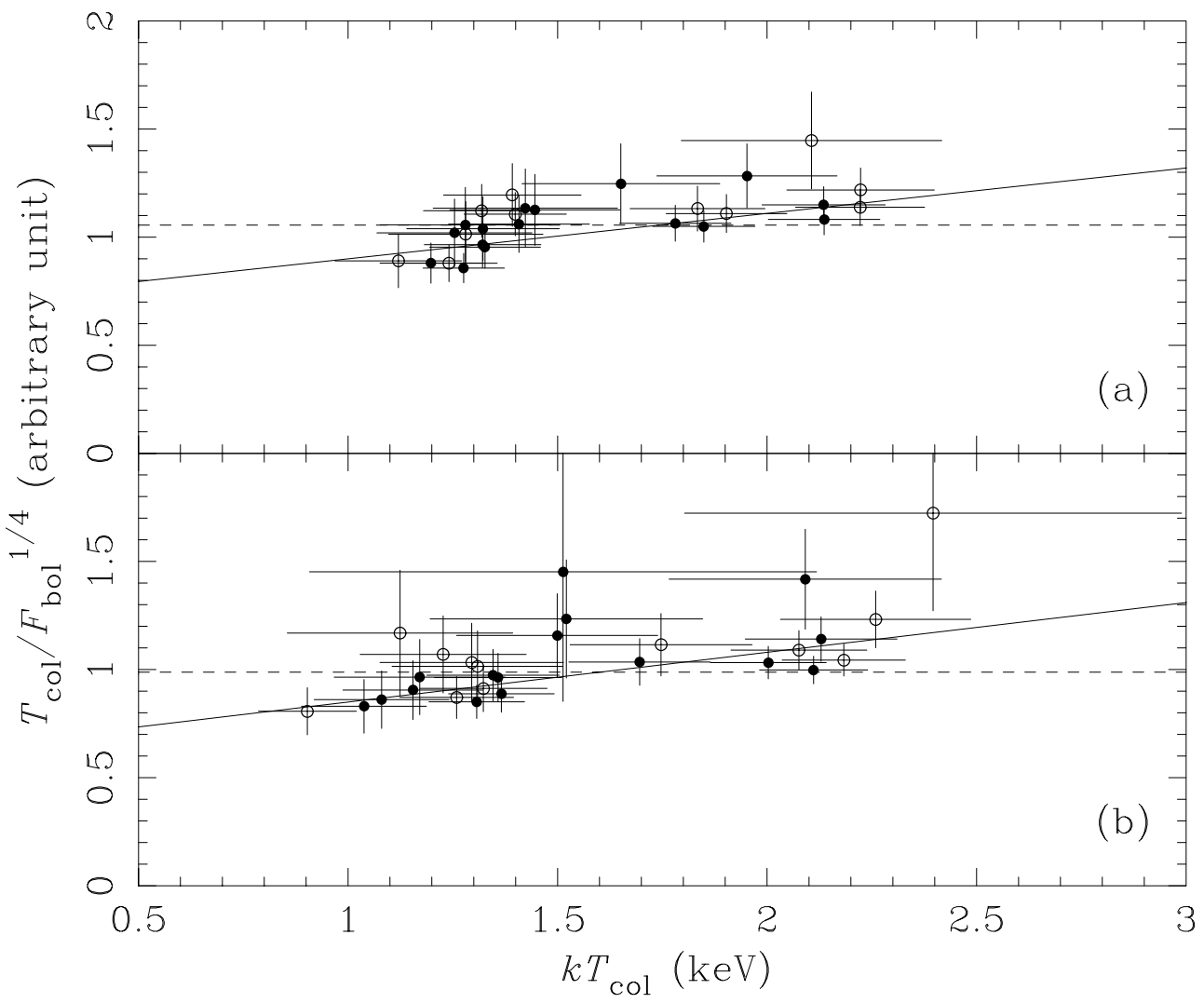

Fig. 9. $T_{\text {col }} / T_{\text {eff }}$ is plotted versus the color temperature $T_{\text {col }}$ a) for the first burst and b) for the second burst of the 1985 observation. Open circles and closed circles represent the values obtained by two different methods, as they do in Fig. 7. Best-fit straight lines are shown as solid lines and straight lines with a constant value as dashed lines for comparision. 
from EXOSAT GSPC observations. However, as White et al. (1986) pointed out, the statistics of the data are insufficient to rule out more complex models. The present analysis yields a better fit with the two component model for the burst source Ser X-1 obtained by ME observations. Seon et al. (1997) argued that the spectra of burst sources are better fitted with a more complex two component model in some cases, at least in the banana state on the color-color diagram. Recently, Church \& Bałucińska-Church (2001) analysed the spectra of Low Mass X-ray Binaries, including burst and non-bursting sources, obtained from ASCA and showed that the Low Mass X-ray Binaries are well-fitted by a two-component emission model. Thus, the spectra of burst sources also should be interpreted, at least in the banana state, using a two-component model, one of which arise from the neutron star surface and the other from the extended accretion disk corona (see also White et al. 1988). The discrepancy of the spectral models between GSPC and ME observations is mainly due to the insufficient statistics of the GSPC observation.

The burst durations are rather short in the present observations, which is typical for Ser X-1 (Sztajno et al. 1983). Sztajno et al. (1983) found a good correlation between the peak flux $F_{\max }$ and the fluence $E_{\mathrm{b}}$ of the burst, with the average burst duration $\tau=6.8 \pm 2.1 \mathrm{~s}$. The values obtained from the present work are also consistent with their results. This short burst duration implies the absence of a significant contribution from the hydrogen burning in these bursts (Fujimoto et al. 1981). The $\alpha$ values obtained also indicate that not only the hydrogen but also helium is strongly burned prior to the bursts (van Paradijs et al. 1988a). Very irregular burst behavior found from $S A S-3$ observations (Li et al. 1977; Sztajno et al. 1983) may be related to the fact that the persistent luminosity is close to a critical value above which $\mathrm{X}$-ray bursts do not occur. We note that the bright burst source XB 1735-444 also shows a very irregular burst behavior (Lewin et al. 1980; van Paradijs et al. 1988b).

The central energy and the equivalent width of the discrete feature observed in the present analysis is consistent with the previously observed $4.1 \mathrm{keV}$ line feature. Waki et al. (1984) identified the $4.1 \mathrm{keV}$ absorption line as resonance absorption of iron $\mathrm{K} \alpha$ transition in He-like iron. However, this interpretation requires an extremely soft equation of state for the nuclear matter, and the large equivalent widths (up to $\sim 500 \mathrm{eV}$ ), which are difficult for theoretical interpretation (see e.g. Foster et al. 1986). Madej (1990) proposed a simple model, in which the features are interpreted as groups of the resonance $\left(\mathrm{K}_{\alpha}\right)$ transitions of He-like and H-like calcium (4.1 keV line) or chromium (5.7 keV line), formed in turbulent circumstellar clouds far from the neutron star surface. More recently, Pinto et al. (1992) pointed out that the problems with the interpretation of the $4.1 \mathrm{keV}$ features as photospheric absorption are avoided if the line is formed in the matter accreting onto a neutron star. Although the detection of the absorption feature is rather marginal in the present analysis, it is worth reporting a possible detection of the $4.1 \mathrm{keV}$ line feature in Ser X-1 burst, as more observational data is required for proper understanding of the discrete feature.

While a simple blackbody provides a reasonable fit to burst spectra from neutron stars, the spectrum is not expected to be perfectly Planckian (see e.g. London et al. 1986; Madej 1997). The model calculation shows that the calculated spectra emerging from the neutron star atmosphere during bursts are harder than the blackbody spectra of the same effective temperature. This spectral hardening effect is attributed to the reduction of the emissivity by electron scattering and the strong frequency dependence of the free-free opacity. The variation of the value $\theta$ as a function of $k T_{\text {col }}$ has been investigated by several authors (see e.g. Penninx et al. 1989 and Damen et al. 1990; more recently Smale 2001), but not for Ser X-1. Bałucińska-Church et al. (2001), however, argue that there is no indication of a departure from simple blackbody spectral shape not only in quiescent spectra of Low Mass X-ray Binaries, but only in burst spectra of burst sources, using the Rossi-XTE PCA with its high sensitivity. They suggested that bursts with $k T \sim 3 \mathrm{keV}$ may have modified spectra, whereas those with $k T \sim 2 \mathrm{keV}$ do not. Kuulkers et al. (2002) also show during burst decay in GX $17+2$ that the blackbody is not modified. The present study of Ser X-1 shows at most marginal evidence of the deviation. More observational data is required for a proper understanding of the spectral feature, since the present study also shows no clear evidence of the deviation from a simple blackbody spectral shape.

In the X-ray light curves of bursts, several types of the multi-peak structures have been recognized. A double-peaked structure, which is accompanied by photospheric radius expansion, occurs in the high-energy part of the spectrum during the very strong burst, while it is not seen in the bolometric luminosity (see e.g. Harberl et al. 1987). Another type is the multipeaked structure found in the bolometric luminosity of some bursts (see e.g. Sztajno et al. 1985). Gottwald et al. (1987) reported a third type of peak structure, which is visible only at low energies and occurs around the peak flux. A double maximum similar to the second type of peak structure is seen from the second burst of the 1985 data in our present analysis. The double peak is not accompanied by photospheric radius expansion, as shown in Fig. 4, although the spectral data has a coarse temporal resolution. Additionally, the double peak is visible in the bolometric luminosity and does not occur at the maximum flux. In this regard, this burst may be similar to relatively weak double-peaked bursts from XB 1636-536 (van Paradijs et al. 1986). We also note that $\mathrm{Li}$ et al. (1977) observed doublepeaked profiles, which are visible in some of the low energy channels in the relatively weak bursts of Ser X-1, although they did not mention anything about the origin of the double-peaked profiles.

Currently, there are two models suggested in an attempt to explain the multiple peak structure seen in the bolometric luminosity. Sztajno et al. (1985) argued that the multi-peaked bursts are the results of variation either in the rate at which thermonuclear energy is released in the flash, or in the rate at which the thermonuclear energy is transported to the surface of the neutron star. A different model was suggested by Melia (1987), in which the multi-peak structure could be the result of scattering of the burst radiation from the material which is induced by the burst itself (burst-induced accretion disk corona). Penninx et al. (1987) argued that the scattering model of the burst-induced accretion disk corona is inconsistent with the observed burst 
properties of XB 1636-536. On the other hand, Penninx et al. (1989) considered the possibility that a double-peaked burst from XB 1608-522 is related to the burst-induced corona.

The double-peaked structure of the present case is more prominent in the low energy band than in the high energy band, as can be seen in Fig. 5. Such a peak structure is similar to that of the burst from XB 1608-522 (Penninx et al. 1989), although the double-peaked feature of XB 1608-522 occurred near the Eddington limit. If the double-peaked structure is related to the burst-induced corona, we would expect that the blackbody temperature just before, during, and just after the dip may not change much. In that case, more prominent double peaks in the low energy band may indicate that not only a scattering corona but also a cold absorber can be generated by the burst itself. On the other hand, if the double-peaked structure is related to the stepwise generation of thermonuclear energy, the blackbody temperature may show a variation similar to the bolometric luminosity. It is worth noting that the double-peaked structure in the blackbody temperature was observed from the bursts of XB 1636-536 (Sztajno et al. 1985). Such a double-peaked structure in the blackbody temperature is not seen in Fig. $7 b$. However, the statistics of the present data are not good enough to give a conclusive test of the model.

Acknowledgements. We thank the anonymous referee for very useful comments.

\section{References}

Asai, K., Dotain, T., Mitsuda, K., et al. 1998, in Hot Universe, Proc. of IAU Symp., 188, ed. K. Koyama, S. Kitamoto, \& M. Itoh (Dordrecht: Kluwer Academic Publishers), 354

Asai, K., Dotani, T., Nagase, F., \& Mitsuda, K. 2000, ApJS, 131, 571

Bałucińska, M., \& Czerny, M. 1985, Acta Astron., 35, 291

Bałucińska-Church, M., Barnard, R., Church, M. J., \& Smale, A. P. 2001, A\&A, 378, 860

Church, M. J., \& Bałucińska-Church, M. 2001, A\&A, 369, 915

Damen, E., Magnier, E., Lewin, W. H. G., et al. 1990, A\&A, 237, 103

Forman, W., Jones, C., Cominsky, L., et al. 1978, ApJS, 38, 357

Foster, A. J., Ross, R. R., \& Fabian, A. C. 1986, MNRAS, 228, 259

Friedman, H., Byram, E., \& Chubb, T. 1967, Science, 156, 374

Fujimoto, M. Y., Hanawa, T., \& Miyaji, S. 1981, ApJ, 246, 267

Gottwald, M., Parmar, A. N., Reynolds, A. P., et al. 1995, A\&AS, 109, 9

Gottwald, M., Stella, L., White, N. E., \& Barr, P. 1987, MNRAS, 229, 395

Hackwell, J. A., Grasdalen, G. L., Gehrz, R. D., et al. 1979, ApJ, 233, L115

Harberl, F., Stella, L., White, N. E., Priedhorsky, W. C., \& Gottwald, M. 1987, ApJ, 314, 266
Hasinger, G., \& van der Klis, M. 1989, A\&A, 225, 79

Kuulkers, J., Homan, J., van der Klis, M., Lewin, W. H. G., \& Mendez, M. 2002, A\&A, 382, 947

Lewin, W. H. G., van Paradijs, J., Cominsky, L., \& Holzner, S. 1980, MNRAS, 193, 15

Li, F. K., Lewin, W. H. G., Clark, G. W., et al. 1977, MNRAS, 179, $21 \mathrm{p}$

London, R. A., Taam, R. E., \& Howard, W. M. 1986, ApJ, 306, 170

Lum K. K., Canizares, C. R., Clark, G. W., et al. 1992, ApJS, 78, 423

Madej, J. 1990, Acta Astron., 40, 223

Madej, J. 1997, A\&A, 320, 177

Melia, F. 1987, ApJ, 315, L43

Nakamura, N., Inoue, H., \& Tanaka, Y. 1988, PASJ, 40, 209

Nishimura, J., Mitsuda, K., \& Itoh, M. 1986, PASJ, 38, 819

Oosterbroek, T., Barret, D., Guainazzi, M., \& Ford, E. C. 2001, A\&A, 366,138

Pinto, P. A., Taam, R. E., \& Laming, J. M. 1992, BAAS, 23, 1320

Penninx, W., Damen, E., Tan, J., Lewin, W. H. G., \& van Pardijs, J. 1989, A\&A, 208, 146

Penninx, W., van Paradijs, J., \& Lewin, W. H. G. 1987, ApJ, 321, L67

Priedhorsky, W. C., \& Terrell, J. 1984, ApJ, 280, 661

Schulz, N. S., Hasinger, G., \& Trümper, J. 1989, A\&A, 225, 48

Seon, K.-I., Min, K.-W., Yoshida, K., et al. 1997, ApJ, 479, 398

Smale, A. P. 2001, ApJ, 562, 957

Swank, J., Becker, R., Pravdo, S., \& Serlemitsos, P. 1976, IAU Circ., 2963

Sztajno, M., \& Bałucińska, M. 1983, Acta Astron., 33, 2

Sztajno, M., Basinska, E. M., Cominsky, R., Marshall, F., \& Lewin, W. H. G. 1983, ApJ, 267, 713

Sztajno, M., van Paradijs, J., Lewin, W. H. G., et al. 1985, ApJ, 299, 487

Sztajno, M., van Paradijs, J., Lewin, W. H. G., et al. 1986, MNRAS, 222,499

Turner, M. J. L., Smith, A., \& Zimmermann, H. U. 1981, Space Sci. Rev., 30, 513

van der Klis, M. 1989, in Timing Neutron Stars, ed. H. Ögelman, \& E. P. J. van der Heuvel (Dordrecht: Kluwer Academic Publishers), 27

van Paradijs, J. 1978, Nature, 274, 650

van Paradijs, J., \& Lewin, W. H. G. 1986, A\&A, 157, L10

van Paradijs, J., Penninx, W., \& Lewin, W. H. G. 1988a, MNRAS, 233, 437

van Paradijs, J., Penninx, W., Lewin, W. H. G., Sztajno, M., \& Trümper, J. 1988b, A\&A, 192, 147

van Paradijs, J., Sztajno, M., Lewin, W. H. G., et al. 1986, MNRAS, 221,617

Vrtilek, S. D., Helfand, D. J., Halpern, J. P. Kahn, S. M., \& Seward, F. D. 1986, ApJ, 308, 644

Vrtilek, S. D., McClintock, J. E., Seward, F. D., Kahn, S. M., \& Wargelin, B. J. 1991, ApJS, 76, 1127

Waki, I., Inoue, H., Koyama, K., et al. 1984, PASJ, 36, 819

White, N. E., Peacock, A., Hasinger, et al. 1986, MNRAS, 218, 129

White, N. E., Stella, L., \& Parmar, A. N. 1988, ApJ, 324, 363 